\title{
Flexible Point Handles Metaphor for Character Deformation
}

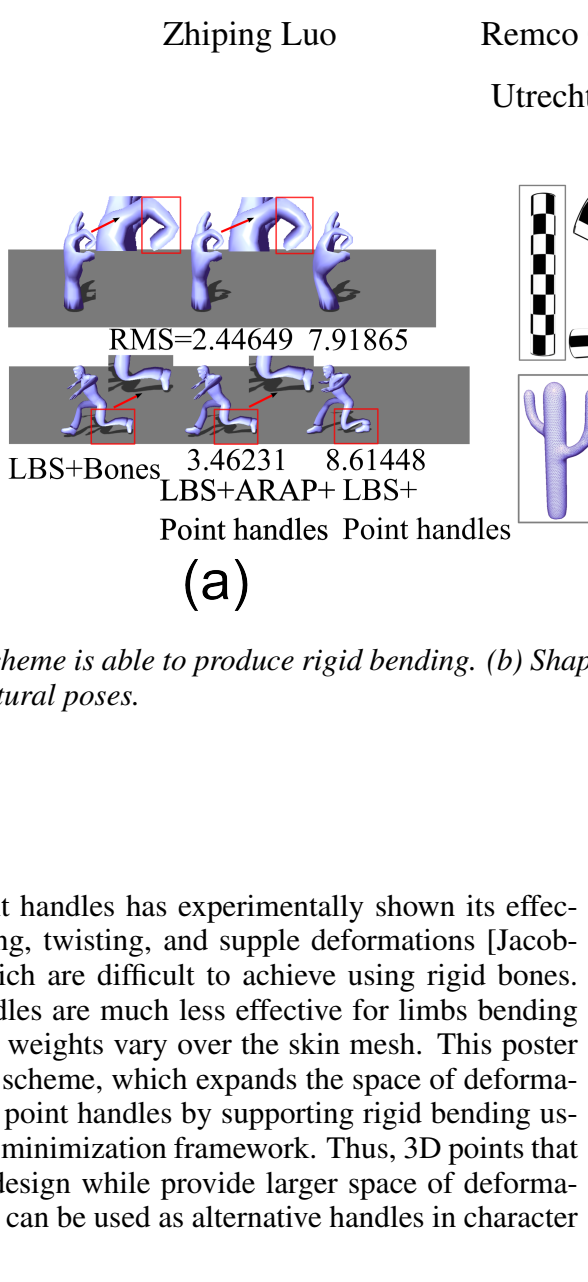

CR Categories: I.3.7 [Computer Graphics]: Three-Dimensional Graphics and Realism-Animation;

Keywords: energy minimization, character skinning, point handles

\section{Point handles metaphor}

Given a model consisting of $H$ point handles, a skin mesh $S$ containing $N$ vertices $\left\{v_{1}, \ldots, v_{N}\right\}$ and $M$ triangles $\left\{t_{1}, \ldots, t_{M}\right\}$ at rest pose, each vertex $v_{i}$ is bound to the handles by influence weights expressed as a vector $\mathbf{w}_{i}=\left(w_{i, 1}, \ldots, w_{i, H}\right)$. Given the transformations of point handles $\left(C_{1}^{f}, \ldots, C_{H}^{f}\right)$ at frame $f$, optimal defor-

Permission to make digital or hard copies of all or part of this work for personal or classroom use is granted without fee provided that copies are not made or distributed for profit or commercial advantage and that copies bear this notice and the full citation on the first page. Copyrights for components of this work owned by others than ACM must be honored. For all other uses, contact the Owner/Author.

Copyright is held by the owner/author(s).

VRST '15, November 13-15, 2015, Beijing, China.

ACM 978-1-4503-3990-2/15/11.

http://dx.doi.org/10.1145/2821592.2821634 mation is computed by solving the minimization problem

$$
\begin{array}{ll}
\min & E_{s}\left(S^{\prime}\right)=\sum_{i=1}^{N} \sum_{j \in \mathcal{N}_{1}\left(v_{i}\right)} \omega_{i, j}\left\|\left(v_{i}^{\prime}-v_{j}^{\prime}\right)-R_{i}\left(v_{i}-v_{j}\right)\right\|^{2} \\
\text { s.t. } & v_{k}^{\prime}=v_{k} \sum_{j=1}^{H} w_{k, j} C_{j}^{f}, \quad k \in \mathcal{H},
\end{array}
$$

where $\mathcal{H}$ is the ${ }^{j=1}$ set of indices of the proximal vertices, $\omega_{i, j}$ are per-edge cotangent weights between $v_{i}$, and each one-ring neighbor $v_{j} \in \mathcal{N}_{1}\left(v_{i}\right) . \quad R_{i}$ of vertex $v_{i}$ is derived from the singular value decomposition (SVD) of the covariance matrix $S_{i}=$ $\sum_{j \in \mathcal{N}_{1}\left(v_{i}\right)}\left(\omega_{i, j} e_{i j} e_{i j}^{\prime T}\right)=U_{i} \sum_{i} V_{i}^{T}$, and $R_{i}=V_{i} U_{i}^{T}$, where $e_{i j}=v_{i}-v_{j}$ and $e_{i j}^{\prime}$ is the edge after reconstruction. The minimization is achieved using the same solution explained in [Sorkine and Alexa 2007].

Results An error metric w.r.t. the ground truth (skeletal deformations) is formulated for quantitative evaluations. The lower the computed value is, the more rigidly the limbs are bent. The metric is calculated as

$$
E_{R M S}=\frac{\sum_{f=1}^{F} \sum_{i=1}^{N}\left\|b v_{i}^{f}-p v_{i}^{f}\right\|^{2}}{\sqrt{3 N F}},
$$

where $F$ denotes the number of frames, $b v^{f}$ and $p v^{f}$ are deformed vertices at frame $f$ resulting from a method with bone weights and point weights, respectively. Our scheme is tested on a human model mainly consisting of rigid bending deformations, and the results shown in Fig. 1(a) demonstrate the outperformance. Closed-form blending techniques, such as Linear Blend Skinning (LBS) and Dual Quaternion Skinning (DQS), do not support shape-reserving property, but our scheme better maintains the shape locality, see Fig. 1(b).

\section{References}

Jacobson, A., Baran, I., Popović, J., And Sorkine, O. 2011. Bounded biharmonic weights for real-time deformation. ACM Trans. Graph. 30, 4 (July), 78:1-78:8.

Sorkine, O., AND AleXA, M. 2007. As-rigid-as-possible surface modeling. In Proceedings of the Fifth Eurographics Symposium on Geometry Processing, SGP '07, 109-116. 